\title{
ABERRATION OF THE FUNCTION
}

\author{
Yoshitomi Tolumitsu, M. D. \\ (Pathological Department, Yonago Med. University)
}

Although there is both a quantative and qualitative change in the function, at present there seems to be greater importance attached to the quantitative changes, minimizing the importance of the qualitative or aberrations of function. For instance, many consider only that the blocking of the reticuloendothelial system brings about hypofunction of the system. The relation with the production of antibodies and with the pathogenesis of jaundice however has given rise to discussion and there are a few men who think of this in relation to the qualitative change of this system.

' In writer's research there are three conditions in which the qualitative changes can be proved.

(1) An unresponsive or paradoxical reaction is developed as a result of an irritation which is expected to show a specific reaction.

(2) The blood of such an animal when injected into a second animal renders the latter unresponsive or results in a paradoxical reaction to the same irritation. Thus it is shown that there is an active substance in the blood of the original animal which is not recognizable in the normal state. This substance is a product of an aberrated organ, because the substance is one resulting in a completely different reaction.

(3) In the normal state, an organ produces two kinds of substance in different amounts and these have an antagonic relation to each other but if a qualitative change is brought about in the function of the organ, the ratio of production of these two substances are reversed. Accordingly in this case the reaction to irritation becomes paradoxical.

Though. by origin, every external cause is able to bring aberration of cells under a definitive condition, generally it is difficult to confirm it concretely. As the result of experiments under the indicator of the foregoing three conditions, writer confirmed that the aberration was caused every time in the following four occasions.

\section{Ex. I. Aberration of the function in generalized disease.}

A disease like syphilis in which the body is universally involved and therefore has definite influence on the various organ gradually and constantly brings the aberration of function to the organ. When a rabbit, infected with syphilis by implantation of a piece of diseased tissue into its testicle is radiated with ultra violet light on its skin, thrombopenia, which is the paradoxical reaction th the normal state, is brought about. In addition thrombopeniogenous substance is produced 
in the body of the rabbit. A paradoxical reaction like this is also caused by the injection of adrenaline or pilocarpine, so that if the blood of a rabbit with the above mentioned management is in jected into another healthy rabbit, thrombopenia is brought about. On the other hand if atropine, which causes thrombopenia in a normal animal, is in jected into a syphilis-rabbit, thrombocytosis (the paradoxical phenomenon) is produced in the rabbits and the thrombocytogenous substance is produced in its blood, especially in the venous blood of spleen. However a splectomized syphilis-rabbit gives a reaction like a normal rabbit to the above mentioned irritation. If the spleen of a normal rabbit is transplanted into a syphilis-rabbit the paradoxical reaction returns to the normal one. From these facts, it must be said that the substance in the blood which causes the paradoxical reaction is produced by the spleen in aberration of function, and that the paradoxical reaction is a phenomenon caused by the aberration of spleen function.

By this time some aberration is present in the kidney. There is an active substance, (called Nephrohormone by Y. Tokumitsu) which has the function of correcting syndromes of kidney disease, in normal venous blood of the kidney. If a body is affected with syphilis, the hormone disappears and another substance (called contranephrohormone by Y. Tokumitsn) with paradoxical function appears in the venous blood of the kidney.

Pertussis vaccine causes lymphocytosis in a healthy animal, but it causes lymph. openia in a syphilis-rabbit and lymphopeniogenous substance appears in its blood. This substance appears in the largest quantity and earliest in the venous blood of liver. Accordingly it is known that syphilis frequently causes aberration of liver function.

About one month after a rabbit is infected with tuberculosis, the quantitative change of thrombocytes shows a paradoxical reaction comparable to that of a normal animal when the rabbit is radiated with ultra violet light or in jected with adrenalin or pilocarpin. The paradoxical reaction is just like the one with syphilis.

\section{Ex. II. Excision of autonomic nerves.}

a) Similar to the syphilis-rabbit, radiation with ultra violet or injection of adrenalin or pilocarpin will cause thrombopenia in an animal in which the splenic nerve has been excited. This paradoxical reaction is again accompanied by an active substance in the blood which can cause thrombopenia in another animal. On the other hand thrombopeniogenous agents, (for example atropine) cause thorombocytosis in this type of animal (the paradoxical reaction) and again this is accompanied by the presence of a thrombocytogenous substance in the blood. These active substances are recognized in greatest quantity and earliest in the spleen and the venous blood of the spleen. Accordingly it must be said that they are the products of the spleen as a result of aberration function. 
b) After the excision of kidney nerves Nephrohormone disappears which existed in the venous of the kidney when it was its normal state, and Contranephrohormone with paradoxical function (consequently plasma chloride increases and blood pressure drops) appears in its venous blood. These phenomena prove that after excision of its nerves, the kidney changes its function qualitatively and produces a chemical substance with paradoxical nature to the normal state. It is especially noteworthy that hypertension caused by amputation of the regulatory nerve of blood pressure (by Hering) recuperates at once following the excision of kidney nerves and that such excision can be used as clinical treatment for essential hypertension.

c) After excision of nervous testicularis, masculine hormone which existed in the vena spermatica interna in the normal state disappears and an active substance with paradoxical nature appears in its blood. By this time every type of seminal epithelial cell except spermatogonia atrophies in the testicle and Laidig's cells show no change.

\section{Ex. III. - Repeated Irritation}

Repeated irritation of cells causes aberration of function very frequently. For instance, after radiation with a definite amount of ultra violet ray on the skin, histamine appears in the blood causing thrombocytosis in the body and stimulating the secretion of much spleen hormone. Following this thrombocytogenous substance is recognized in the blood. If the radiation is repeated for more than four weeks, histamin continues to increase in the blood but instead of thrombocytogenous substance, thrombopeniogenous substance appears resulting in a decrease of thrombocytes. The reason for these phenomena is that repeated exposure to histamine results in an aberration of function of the spleen. Repeated irritation by photodynamic action also leads to the same paradoxical phenomena in the number of leucocytes, but the pathogenesis is very different from one caused by repeated radiation with ultra violet ray. Normally the spleen secretes two kinds of active substances in its venous blood. One of these is leucocytogenous substance, a lipid, which is secreted in large quantities, and the other, leucopeniogenous substance a protein, which is secreted in small quantities.

Exposure to the sunlight after injection of a fluorescent substance in venous blood causes remarkable leucocytosis and a large quantity of leucocytogenous substance is produced in the blood especially in the venous blood of the spleen. If this irritation is repeated for more than three weeks, leucopenia appears gradually and leucopeniogenous substance is produced in the blood.

The ratio of these two substances (that is, the lipid and the protein) in venous blood of spleen reverses. After splenectomy the number of leucocytes is not changed by the same photodynamic action. The above mentioned paradoxical phenomenon results from the increase of leucopeniogenous substance from the spleen which 
is extremely small in the normal state. This also is an example of an aberration of functional activity. The reticuloendothelial system also changes its function qualitatively when irritation is repeated.

Generally it is thought that the phenomenon of reticuloendothelial system blocking, resulting from the injection of solid bodies or india ink into the blood, is a hypofunction of the system, but according to the writer's experiment the phenomenon is an aberration of function. The following facts are the proof for this statement. If a small amount of india ink is injected into the venous blood of a rabbit, the resistance to strychinine, morphine, and taurocholic acid is inereased and a substance appears in the blood which is capable of diminishing the toxic effect of these poisons, of course, this phenomenon comes from hyperfunction of the R. E. S. On the other hand, if the same quantity of india ink is injected once a day for four days, the rabbit's resistance to those poisons greatly diminishes.

At the present time it is generally thought that the phenomenon is the result of hypofunction of the R. E. S., but according to writer's experiments the Phenomenon is not caused by hypofunction alone. The reason is as follows: blocking caused by the repeated injection of india ink results in the presence of an active substance in the blood which weakens the resistance to the poisons in another body. Moreover the substance has a paradoxical function to the substance resulting from irritation of the reticulo-endothelial system. Further it is definite that the substance is a product of the blocked reticuloendothelial system. In other words the blocking of the R. E. S. causes not only its hypofunction but also the formationof a new product as a result of qualitative change of function.

The foregoing statements are supported by experiments on antibody production in the R. E. S.

The repeated injection of an organic emulsion or organic autolysate causes aberration of functicn in the same organ. For instance, repeated injection of a kidney extract causes disappearance of nephro-hormone in the venous blood of the kidney and the production of contra-nephrohormone.

When testicular extract is injected, the masculine hormone disppears and a substance which causes atrophy of the comb appears.

Injection of a spleen extract causes a severe anemia and a substance is produced which causes anemia in another animal. The substance which causes anemia is not produced in a splenectomized animal. Accordingly it can be said that the substance is the product of the spleen with aberration of function.

The parotid gland is an organ which secretes a hormone in its venous blood capable of decreasing the blood calcium. The repeated injection of parotid gland extracts causes, on the contrary, a hypercalcemic effective substance in its venous blood.

As shown in the above experiments, repeated irritation by injection of an 第26卷 第7號 
organ extract causes aberration of function of the organ from which the extract has been made.

In jection of $\mathrm{CaCl}_{2}$ solution invites fever but repeated injection causes hypothermia. It was proved by the above mentioned manipulation that this phenomenon is a result of the aberration of the parathyroid glands.

Injection of 2.Ccc per $\mathrm{kg}$. $10 \% \mathrm{NaCl}$ solution brings a rise of temperature to the experimental animal, but this treatment repeated for more than two weeks brings no change of temperature. If the blood of a rabbit which has repeatedly been injected, is injected into another rabbit, no change of temperature results from the first injection of $\mathrm{NaCl}$. If the thyroid gland is removed, the active substance in its blood disappears and when the thyroid gland is implanted in other normal rabbit, the paradoxical reaction is brought about. Accordingly it must be said that such abnormal reaction comes from the aberration of thyroid function.

\section{Ex. IV. Extirpation of Endocrine Organs.}

In many cases the extirpation of an endocrine organ causes hyperfunction of another organ which has a close correlation with the excised one. Also it frequently causes aberration of the remaining organ.

For instance, a hypophysectomized animal has a markedly diminished ability to produce antibodies and also has an active substance in its blood stream capable of diminishing antibody production in another animal. This substance is the same as the one produced when the reticulo-endothelial system is blocked. Accordingly it seems that hypophysectomy causes the aberration of the reticuloendothelial system.

After extirpation of its parathyroid glands, the rabbit produces an active substance which controls monocytosis coming from various causes. As the substance is recognized in greatest quantity and earliest in the venous blood of spleen, and moreover disappears after the extirpation of spleen, the substance seems to be a product originating in the aberrated spleen.

The pinealectomized rabbit shows an abnormal salt fever. For example, the temperature of a pinealectomized rabbit descends after injection of $1.0 \mathrm{cc}$ per $\mathrm{kg}$. of $5 \%$ calcium chloride solution while a normal animal's ascends. Similàrly the temperature ascends after injection of $1.0 \mathrm{cc}$ per $\mathrm{kg}$. of $5 \% \mathrm{NaCl}$ while a normal animal shows no reaction. There is a substance in the blood of pinealectomized rabbits which causes abnormal salt-fever when in jected into normal rabbits.

If after pinealectomy, the thyroid gland is removed, the abnormal salt-fever caused by $\mathrm{NaCl}$ disapoears, and if its parathyroid glands are removed, the abnormal salt-fever caused by $\mathrm{CaCl}_{2}$ disappears. When the thyroid gland or parathyroid gland of the pinealectomized rabbit is implanted in other normal rabbit, the abno. rmal salt-fever for $\mathrm{NaCl}$ or $\mathrm{CaCl}_{2}$ solution is brought about, due to the implantation 
of those organs (the thyroid or parathyroid gland) of a normal rabbit; however, such abnormal salt-fever does not appear. That is to say, the cause of the abnormal fever by the above mentioned two salts is secondary, coming from the aberration of the thyroid and parathyroid function.

From the above mentioned experiments, it can be said that the change in function is not only quantitative, but very frequently also qualitative.

The verification of this statement is frequently accompanied with difficulty. The objective, however, can be attained by finding an active substance in the blood and by investigating the organ which produces the substance.

In conclusion I want to emphasize the followiug two matters. One is, when an irritation is repeated, the irritated body or part gradually becomes insensible to the irritation and this is called "accustoming." The "accustoming" seems to be a kind of functional aberration of the irritated tissue, and is the phenomenon which is seen everybody. It may be called physiological aberration. The other is, when the function of some organ is aberrated as in some of the foregoing experiments, the body shows no reaction or paradoxical reaction to various drugs, (for example, adrenalin, pilocarpin, atropine, etc.) in many cases. These are the phenomena which are not explainable in the light of present pharmacology which deals with the mechanism of drugs with regard to healthy bodies. On the other hand our concern is the patient, and since it would not be at all unusual for a patient to have an aberrated organ, a considerable number of drugs should be re-examined with special. reference to pathological pharmacology.

\section{Conclusion}

The pathological change of function of an organ is not a quantitative alone. A knowledge of the pathogenesis and the method of medical treatment is very difficult unless the qualitative changes are also investigated. 